\title{
Genome-wide dissection of globally emergent multi-drug resistant serotype I9A Streptococcus pneumoniae
}

\author{
Dylan R Pillai* ${ }^{* 1,2,3}$, Dea Shahinas ${ }^{\dagger 1}$, Alla Buzina ${ }^{2}$, Remy A Pollock ${ }^{2}$, \\ Rachel Lau2 ${ }^{2}$ Krishna Khairnar ${ }^{2}$, Andrew Wong2, David J Farrell1,2, \\ Karen Green ${ }^{3}$, Allison McGeer ${ }^{1,3}$ and Donald E Low ${ }^{1,2,3}$
}

Address: ${ }^{1}$ Department of Laboratory Medicine and Pathobiology, University of Toronto, ON, Canada, ${ }^{2}$ Ontario Agency for Health Protection and Promotion, Toronto, ON, Canada and ${ }^{3}$ University Health Network/Mount Sinai Hospital, Toronto, ON, Canada

Email: Dylan R Pillai* - dylan.pillai@oahpp.ca; Dea Shahinas - dea.shahinas@oahpp.ca; Alla Buzina - alla.buzina@ontario.ca; Remy A Pollock - remy.pollock@ontario.ca; Rachel Lau - rachel.lau@oahpp.ca; Krishna Khairnar - krishna.khairnar@oahpp.ca; Andrew Wong - nderoo.wong@gmail.com; David J Farrell - david.farrell@ontario.ca; Karen Green - kgreen@mtsinai.on.ca;

Allison McGeer - amcgeer@mtsinai.on.ca; Donald E Low - dlow@mtsinai.on.ca

* Corresponding author †Equal contributors

Published: 30 December 2009

BMC Genomics 2009, 10:642 doi:10.1 |86//47|-2/64-10-642
Received: 16 September 2009

Accepted: 30 December 2009

This article is available from: http://www.biomedcentral.com//47/-2/64//0/642

(c) 2009 Pillai et al; licensee BioMed Central Ltd.

This is an Open Access article distributed under the terms of the Creative Commons Attribution License (http://creativecommons.org/licenses/by/2.0), which permits unrestricted use, distribution, and reproduction in any medium, provided the original work is properly cited.

\begin{abstract}
Background: Emergence of multi-drug resistant (MDR) serotype 19A Streptococcus pneumoniae (SPN) is well-documented but causal factors remain unclear. Canadian SPN isolates (1993-2008, $n$ $=1 \mathrm{I}, 083)$ were serotyped and in vitro susceptibility tested. A subset of MDR I9A were multi-locus sequence typed (MLST) and representative isolates' whole genomes sequenced.

Results: MDR 19A increased in the post-PCV7 era while 19F, 6B, and 23F concurrently declined. MLST of MDR 19A $(n=97)$ revealed that sequence type (ST) 320 predominated. ST320 was unique amongst MDR 19A in that its minimum inhibitory concentration (MIC) values for penicillin, amoxicillin, ceftriaxone, and erythromycin were higher than for other ST present amongst postPCV7 MDR 19A. DNA sequencing revealed that alleles at key drug resistance loci $p b p 2 a, p b p 2 x$, pbp2b, ermB, mefA/E, and tet $M$ were conserved between pre-PCV7 ST $32019 \mathrm{~F}$ and post-PCV7 ST 320 19A most likely due to a capsule switch recombination event. A genome wide comparison of MDR 19A ST320 with MDR I9F ST320 identified 822 unique SNPs in 19A, 61 of which were present in antimicrobial resistance genes and 100 in virulence factors.
\end{abstract}

Conclusions: Our results suggest a complex genetic picture where high-level drug resistance, vaccine selection pressure, and SPN mutational events have created a "perfect storm" for the emergence of MDR I9A.

\section{Background}

The introduction of the heptavalent polysaccharide capsule vaccine (PCV7; serotypes 4, 6B, 9V, 14, 18C, 19F, and 23F) in North America as a pediatric universal vaccine program has led to a significant decrease in vaccine sero- type invasive pneumococcal disease (IPD) [1] and drugresistant pneumococci. However, reports of the vaccine's success have been tempered by observed increase in the prevalence of non-vaccine serotypes (NVS) and in particular the multi-drug resistant (MDR) NVS 19A [2-11]. 
Multi-locus sequence typing (MLST) of these isolates has demonstrated expansion of certain clonal complexes (CC). The genetic determinants that are driving the success of certain CC remain poorly defined. Several plausible and possibly overlapping hypotheses have been suggested. The first suggests that MDR 19A is a result of a genetic recombination event resulting in "capsule switch" $[12,13]$, thereby giving it a fitness advantage over other vaccine serotypes by not being subjected to immune selective pressure. Second, MDR 19A existed before the implementation of PCV7 and has simply replaced vaccine serotypes (VS) targeted by PCV7 [14]. Third, MDR 19A has attained conserved genetic markers which confer resistance to antibiotics commonly used in the treatment of Streptococcus pneumoniae (SPN) invasive pneumococcal disease (IPD) [15]. These genetic markers are missing in the drug susceptible SPN 19A isolates, such as those belonging to sequence type 199. Chief amongst these antibiotics conferred resistance to, are $\beta$-lactams and macrolides. Resistance to these drugs likely confers a fitness advantage to the organism at the population level where antibiotics are commonly dispensed [15]. Of note, highlevel penicillin resistance has been mainly associated with serotypes 6A, 6B, 9V, 14, 19A, 19F, and 23F [1]. Successful expansion of MDR 19A SPN in the post-PCV7 introduction era poses a serious global public health risk. The heptavalent pneumococcal conjugate vaccine (PCV7, Prevnar, Wyeth, Madison, NJ) was approved mid-2001 for use in children in Canada [16]. By early 2006, all provinces and territories in Canada had instituted this vaccine into their routine vaccination programs. We describe here the results of comparative genomics of the emerging multidrug resistant serotype 19A identified from 15 years of SPN serotype and susceptibility testing surveillance in relation to PCV7 introduction in Canada.

\section{Results}

\section{Serotype surveillance and susceptibility testing of MDR SPN in the Canadian Bacterial Surveillance Network (CBSN) 1993-2008}

Serotypes and antibiotic susceptibility testing were determined for $n=11,083$ isolates over a 15 year period. Serotype surveillance demonstrated a reduction in vaccine serotypes from the era immediately prior to PCV7 introduction in Canada (pre-PCV7, 1993-2001), PCV7 introduction era (2002-2005), and post-PCV7 introduction era (2006-present) (our unpublished data). We focus here on the MDR serotypes. Figure 1 graphically depicts the trends in MDR (defined as non-susceptibility to penicillin plus two other antibiotics) serotypes $6 \mathrm{~B}, 23 \mathrm{~F}, 19 \mathrm{~F}$ and $19 \mathrm{~A}$, the major contributors to MDR SPN in this population as a percent of all MDR isolates collected for that year. While other serotypes contribute to MDR, their numbers were not significantly large in our database.

\section{Multi-locus sequence typing (MLST) of MDR I9A SPN isolates}

Due to the rising absolute number of MDR 19A isolates, MLST was performed to establish the genetic background of these strains. Figure 2 demonstrates that sequence type (ST) 320, part of CC271, accounts for the majority of MDR 19A following the introduction of PCV7 (postPCV7) in Canada. Prior to PCV7 introduction, ST320 was most significantly associated with serotype $19 \mathrm{~F}$ in this study (our unpublished data). ST320 is a single-locus variant (different at one gene in the MLST schema comprising seven genes) of Taiwan 19F-14 (ST236) which spread globally in the pre-PCV7 era [17]. Categorical clustering of MDR 19A based on MLST demonstrated that ST320 was associated with high-level penicillin resistance (minimum inhibitory concentration/MIC $\geq 4 \mu \mathrm{g} / \mathrm{mL}$ ) (Figure 3). In contrast, non-MDR 19A control isolates taken from CBSN were associated with different STs (Figure 4). Pre-PCV7 MDR 19F high-level penicillin resistance was also strongly associated with ST320 in our Canadian database (Figure 5). Table 1 to 5 summarize actual MIC values for individual MDR 19A isolates by ST. MIC values for penicillin (Table 1), ceftriaxone (Table 2), amoxicillin (Table 3), erythromycin (Table 4), and ciprofloxacin (Table 5) are generally higher for ST320 when compared to other STs amongst MDR 19A. Although not statistically significant, the most notable trends among these antibiotics were penicillin and amoxicillin where ST320 was high-level resistant, while other STs amongst MDR 19A were less resistant or susceptible. Additional file 1http://www.pil lailab.com/suppdata/index.html shows eBURST results and summary statistics for all MLST carried out in this study. Novel MLST are detailed in Additional file $2 \underline{\mathrm{http}}: / /$ www.pillailab.com/suppdata/index.html.

\section{Analysis of antibiotic resistance alleles by DNA sequencing} Mutations associated with elevated minimal inhibitory concentrations (MIC) have been described for key antibiotics commonly used to treat both invasive and non-invasive SPN infection $[1,15,18,19]$. DNA sequence analysis for key resistance-conferring residues in penicillin binding proteins $(p b p) 2 x, 1 a$, and $2 b$ showed conservation between MDR 19A (this study), MDR 19F (this study), and MDR 19A from US and Korea (Table 6). However, residues $339(\mathrm{~F})$ and $400(\mathrm{~T})$ of $\mathrm{Pbp} 2 \mathrm{x}$ were unique to a representative MDR 19A in the US and have been associated with high-level ceftriaxone resistance not seen in Canadian isolates $[10,20]$. Susceptible $19 \mathrm{~A}$ isolates lacked mutations at key residues in $p b p$ genes which are associated with $\beta$ lactam resistance [14]. Similarly, MDR 19A and $19 \mathrm{~F}$ had complete conservation at residues of ermB, mefA, mef $E$ and tetM (all located on the transposon Tn2010) associated with resistance to macrolides and tetracyclines [15]. Identical mutations in Tn2010 were also observed for representative US and Korea MDR 19A iso- 


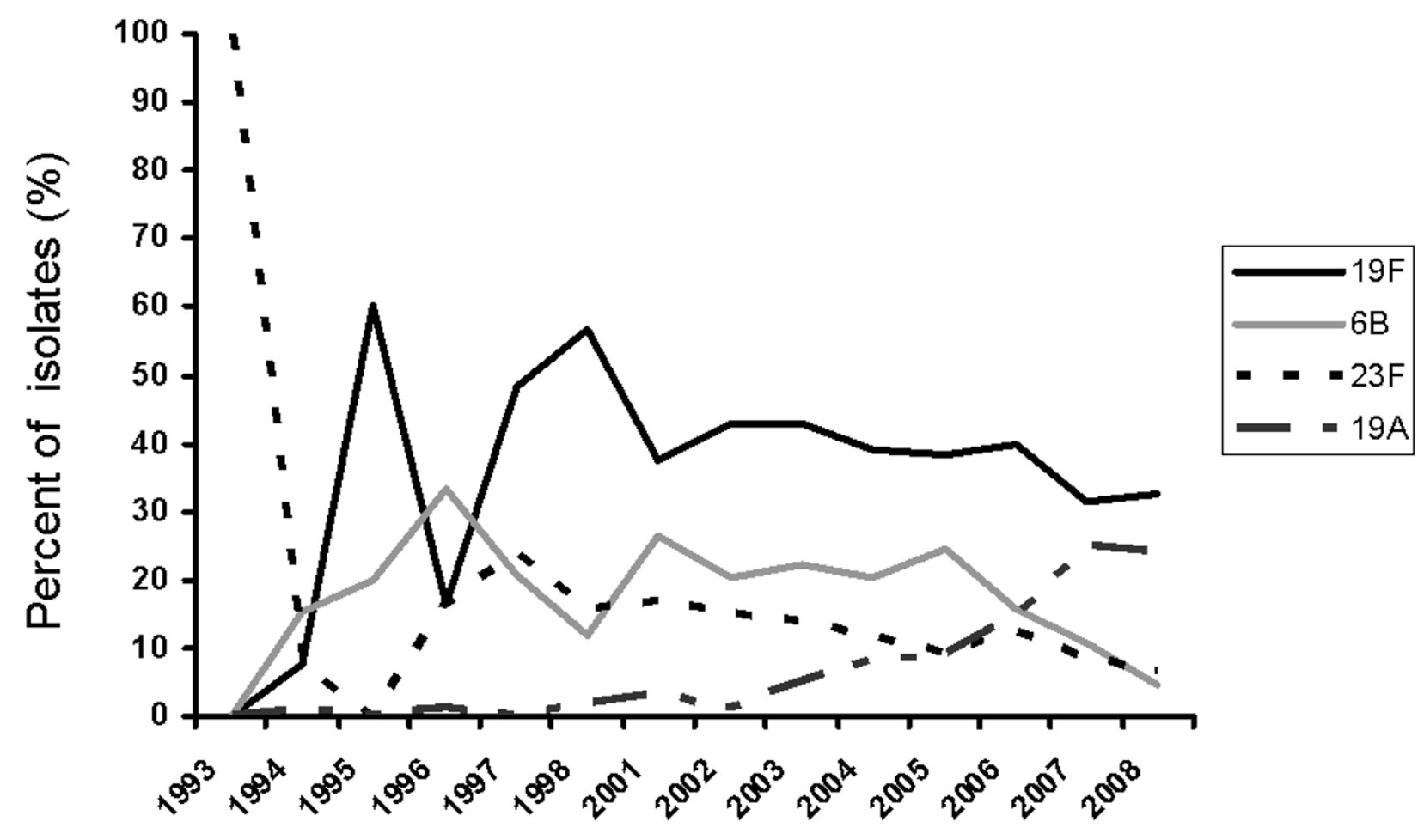

Year

\section{Figure I}

Serotype trends amongst multi-drug resistant (MDR) strains obtained from the Canadian Bacterial Surveillance Network between 1993 and $2008(n=I$ I,083). MDR I9F $(n=477), 23 F(n=150)$, and 6B $(n=22$ I) emerged in the pre- PCV 7 introduction era (before 200I) and continued to rise during vaccine introduction (2002-2005), then declined in post-PCV7 introduction era (2006 onwards). MDR 19A $(n=97)$ was present in the pre-PCV7 at very low levels and began to rise soon after PCV7 was introduced country-wide. Data for 1999 and 2000 were not collected. Data are presented as the percent of all MDR isolates collected for the given year.

lates. Genome insertion points of Tn2010 were heterogeneous amongst MDR 19A and 19F based on polymerase chain reaction (PCR) using primers derived from whole genome sequencing. Additional file 3http://www.pil lailab.com/suppdata/index.html provides the data set for all drug resistance gene sequences and Tn2010 insertion site confirmation results by PCR.

\section{Whole genome sequence of MDR I9A ST320 from the post-PCV7 introduction era}

A representative isolate of SPN MDR 19A ST320 (Ontario, 2007, sputum isolate from patient with pneumonia) from the period immediately after universal coverage of PCV7 was identified for whole genome sequencing (WGS). Figure 6 summarizes the whole genome and each locus can be navigated in Additional file 4http://www.pil lailab.com/suppdata/index.html. MDR 19F ST320 from the pre-PCV7 introduction era (Ontario, 2001, blood isolate from patient with sepsis) was also sequenced using the same method. Sequence comparison of $19 \mathrm{~F}$ and $19 \mathrm{~A}$ ST320 genomes, sequenced and identified $0.41 \%$ difference in AT content and $0.06 \%$ difference in GC content for an overall shared identity of $99.7 \%$ between the two genomes. No evidence of large insertions and deletions between the two genomes was identified.

Figure 7 provides the breakdown of SNPs unique to MDR 19A ST320. When compared to MDR 19F ST320 and reference strain R6 (Genbank AE007317), 822 unique SNPs were identified in the genome of MDR 19A, 169 of which were non-synonymous [21]. Compared to another serotype 19A WGS in Genbank (Hungary 19A-6, NC 010380, 1989, non-invasive), 9484 SNPs were identified. Of the 822 unique SNPs, 61 SNPs (7.4\%) were identified in 


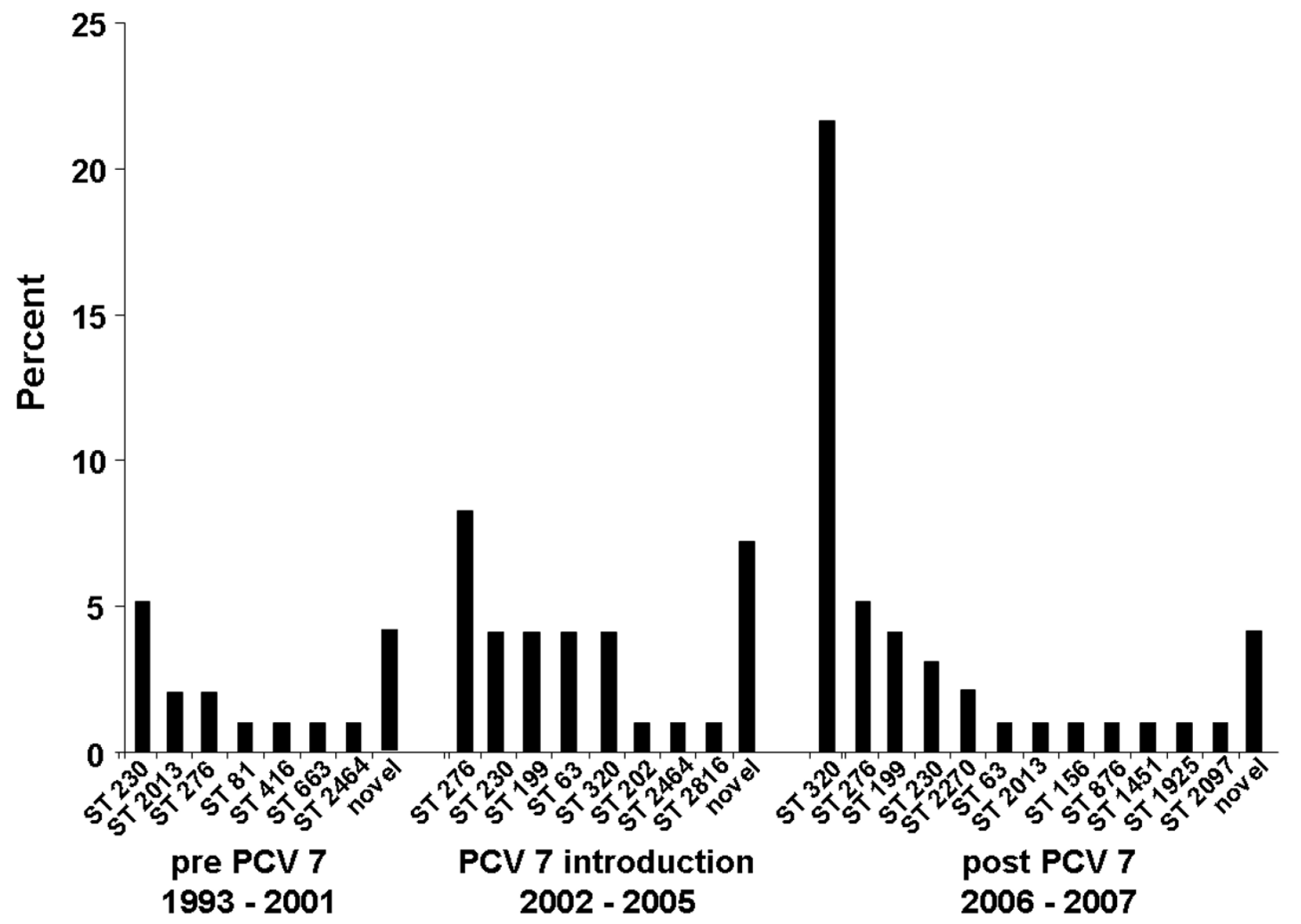

Figure 2

Multi-locus sequence typing (MLST) of multi-drug resistant (MDR) serotype I9A isolates (n = 97) obtained from the Canadian Bacterial Diseases Surveillance Network during pre-PCV 7 introduction, vaccine introduction, and post-vaccine introduction eras. Sequence types (ST) are depicted as a percent of all MDR I9 isolates for that period. ST320 has emerged as the singular dominant sequence type amongst MDR I9A isolates in the post-PCV7 era. Novel implies a collection of strains for which no sequence type (ST) was identified within the MLST http://www.mlst.net/ database at this time but submissions have been made and are summarized in Additional file 2. eBURST summary data for MDR I9A, MDR I9F $(n=30)$ and susceptible I9A $(n=19)$ controls are appended in Additional file Ihttp://www.pillailab.com/suppdata/ index.html.

genes associated with drug resistance and 100 SNPs (12.1\%) in virulence factors. A further 14,208 SNPs were shared between MDR 19A and 19F ST320, whereas 1536 SNPs were unique to $19 \mathrm{~F}$. Of the 61 unique mutations in antimicrobial resistance genes for MDR 19A, seven were in the $p b p$ gene family associated with $\beta$ lactam resistance, one was in the $\operatorname{crcB}$ family associated with fluoroquinolone resistance, and 53 were in the folate pathway genes associated with sulfa drug resistance. Unique SNPs in virulence genes included surface protein pspA precursor ( $n=11$ SNPs identified in MDR 19A compared to reference strain R6), determinant for enhanced expression of pheromone $(n=4)$, hyaluronate lyase precursor $(n=4)$, unsaturated glucuronyl hydrolase $(n=2)$, type 2 capsule locus of SPN $(n=39)$, choline-binding protein F $(n=5)$, choline binding protein $\mathrm{A}(n=11)$, histidine kinase $(n=$ $3)$, toxin expression - transcriptional accessory protein $(n$ $=4)$, pneumococcal histidine triad protein $\mathrm{D}$ precursor $(n$ =3), immunoglobulin A1 protease $(n=11), N$-acetylneuraminate lyase subunit $(n=2)$, and sialidase B precursor (neuraminidase B) $(n=1)$. Additional file 5http:// www.pillailab.com/suppdata/index.html details the gene annotation of unique and shared SNPs (synonymous versus non-synonymous) for MDR 19A and 19F, genome location, and functional category. Summary statistics of the whole genome sequencing can be obtained in Addi- 


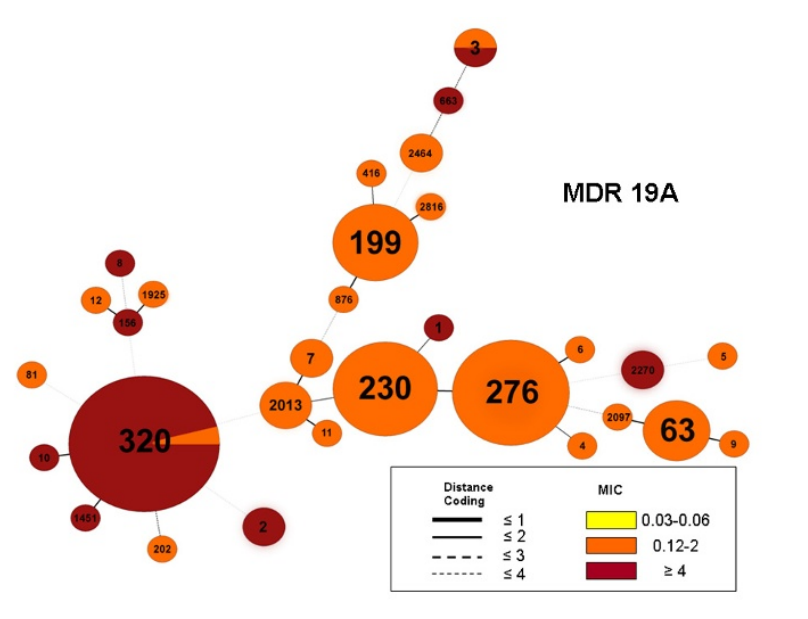

Figure 3

Minimum spanning tree of multi-drug resistant (A) (MDR) serotype I9A $(n=97)$ using BioNumerics software. A categorical clustering was performed based on multi-locus sequence type (MLST). Sequence types sharing the maximum number of single-locus variants were connected first. Each circle represents a sequence type (ST) the size of which is proportional to the number of isolates within that particular ST. Colors within circles indicate the minimum inhibitory concentration (MIC) ranges for penicillin. Relationships between the STs are depicted by the lines connecting the STs and the relative lengths of the branches linking them. Distance coding enumerates the number of differences at a given MLST locus. A distance coding of greater than 2 implies a different clonal complex. Angles of the line connections and the overlapping circles have no significance.

tional files 6 and 7http://www.pillailab.com/suppdata/ index.html.

\section{Capsule locus sequencing analysis}

DNA sequence comparison was carried out for the capsule locus. Figure 8 depicts the arrangement of genes flanking the capsule locus which are similar to previously published data with some notable exceptions [22,23]. For example, A 1277 base pair (bp) region encoding the $\mathrm{ABC}$ co-transporter of the vex operon was present adjacent to the capsule locus in post-PCV7 MDR 19A ST320 but not in MDR 19F. The vex operon in its entirety was located outside of the capsule locus for MDR 19F. The vex gene has homology to $\mathrm{ABC}$ co-transporters and has been linked to vancomycin tolerance in SPN [24]. Of note, $\beta$ lactam resistance genes $p b p 2 x$ and $p b p 1 a$ were adjacent to the capsule locus and, as summarized in Table 6, harboured key mutations that confer resistance to $\beta$ lactam drugs. Alignment of $p b p 1 a$ and $p b p 2 x$ gene sequences from representative ST320 MDR 19A (progeny, $n=3$ ), ST320 MDR 19F (putative recipient, $n=3$ ), and ST199 19A (putative donor, $n=2$ ) demonstrated one recombination point

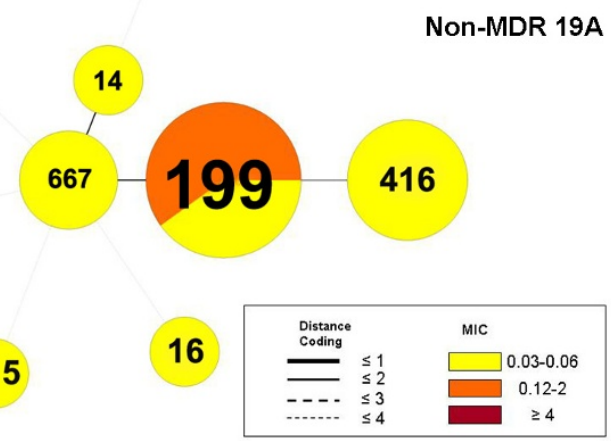

Figure 4

Minimum spanning tree of multi-drug resistant susceptible I9A control isolates $(n=16)$ using BioNumerics software. A categorical clustering was performed based on multi-locus sequence type (MLST). Sequence types sharing the maximum number of single-locus variants were connected first. Each circle represents a sequence type (ST) the size of which is proportional to the number of isolates within that particular ST. Colors within circles indicate the minimum inhibitory concentration (MIC) ranges for penicillin. Relationships between the STs are depicted by the lines connecting the STs and the relative lengths of the branches linking them. Distance coding enumerates the number of differences at a given MLST locus. A distance coding of greater than 2 implies a different clonal complex. Angles of the line connections and the overlapping circles have no significance.

located within $p b p 2 x$ and the other identified by genome walking distal to pbp1a (Figure 8 and Additional file 8http://www.pillailab.com/suppdata/index.html). In contrast to $p b p 2 x$ where homologous and heterologous SNPs occur, pbp $1 a$ showed perfect conservation between recipient and donor strains.

\section{Discussion}

Serotype surveillance of MDR strains in the CBSN database confirmed what other countries have observed - specifically the increased prevalence of MDR 19A, with a concomitant decline in MDR serotypes included in the PCV7 (6B, 23F, and 19F in our study). MLST analysis of MDR 19A was able to identify ST320 as the dominant emerging genotype. This was in contrast to previous studies $[10,13]$ which demonstrated clonal expansion of an existing ST199 MDR 19A after PCV7 introduction but in agreement with other groups $[7,8,25]$. ST320 appears to have higher than usual resistance to commonly used antibiotics such as penicillin, amoxicillin, and ceftriaxone based on MIC values and is challenging to treat clinically especially in the case of bacterial meningitis $[25,5]$. This 


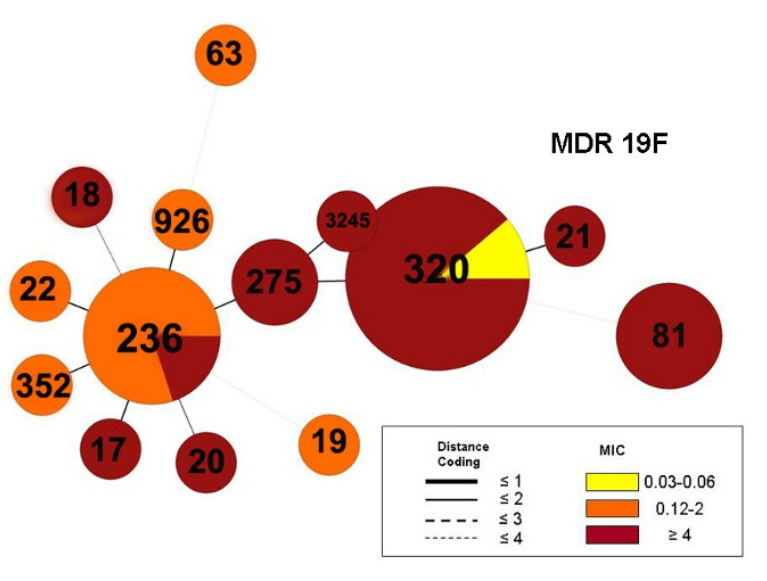

Figure 5

Minimum spanning tree of multi-drug resistant MDR I9F isolates $(n=29)$ from the pre-PCV7 era using BioNumerics software. A categorical clustering was performed based on multi-locus sequence type (MLST). Sequence types sharing the maximum number of single-locus variants were connected first. Each circle represents a sequence type (ST) the size of which is proportional to the number of isolates within that particular ST. Colors within circles indicate the minimum inhibitory concentration (MIC) ranges for penicillin. Relationships between the STs are depicted by the lines connecting the STs and the relative lengths of the branches linking them. Distance coding enumerates the number of differences at a given MLST locus. A distance coding of greater than 2 implies a different clonal complex. Angles of the line connections and the overlapping circles have no significance.

may explain in part ST320 expansion under drug selection pressure. The same genotype and phenotype were present in MDR $19 \mathrm{~F}$ in the pre-vaccine era - the major contributor to ST320 prior to PCV7 introduction in our study. This was not surprising as 19F ST320 is a single locus variant of Taiwan 19F-14 which spread globally in the pre-PCV7 era [26-28]. It was logical then to test the hypothesis that

Table I: Penicillin minimum inhibitory concentration (MIC, top row in bold, $\mu \mathrm{g} / \mathrm{mL}$ ) values for multi-drug resistant (MDR) serotype 19A by sequence type (ST) $(n=97)$.

\begin{tabular}{llllllllll}
\hline & \multicolumn{3}{c}{ S } & \multicolumn{3}{c}{$\mathbf{R}$} & & \multicolumn{1}{c}{ HR } \\
ST & $\mathbf{0 . 0 3}$ & $\mathbf{0 . 0 6}$ & $\mathbf{0 . 1 2}$ & $\mathbf{0 . 2 5}$ & $\mathbf{0 . 5}$ & $\mathbf{I}$ & $\mathbf{2}$ & $\mathbf{4}$ & $\geq 8$ \\
\hline 63 & & & 4 & 1 & & & & & \\
199 & 2 & & 11 & 1 & & & & & \\
416 & 3 & & & & & 1 & & & \\
230 & & & & 2 & 8 & 2 & & & \\
276 & & & & & 1 & 12 & 2 & & \\
320 & & & & & & & 1 & 22 & 2 \\
2013 & & & & 1 & 2 & & & & \\
\hline
\end{tabular}

S, susceptible; I, intermediate; R, resistant; HR, highly resistant.
Table 2: Ceftriaxone minimum inhibitory concentration (MIC, top row in bold, $\mu \mathrm{g} / \mathrm{mL}$ ) values for multi-drug resistant (MDR) serotype I9A by sequence type (ST) $(n=97)$.

\begin{tabular}{llllllll}
\hline ST & $\mathbf{0 . 0 3}$ & $\mathbf{0 . 0 6}$ & $\mathbf{0 . 1 2}$ & $\mathbf{0 . 2 5}$ & $\mathbf{0 . 5}$ & $\mathbf{I}$ & $\begin{array}{l}\mathbf{R} \\
\mathbf{6}\end{array}$ \\
\hline 3 & & & & 5 & & & \\
199 & 1 & 4 & $\mathrm{I}$ & 8 & & & \\
416 & 3 & & & & & $\mathrm{I}$ & \\
230 & & & 2 & 9 & & & \\
276 & & & & & 2 & II & 2 \\
320 & & & & & 1 & I & 23 \\
2013 & & & & 3 & & & \\
\hline
\end{tabular}

S, susceptible; I, intermediate; R, resistant; HR, highly resistant.

MDR 19A ST320 emerged from pre-existing MDR 19F ST320. To lend credence to this hypothesis, it was noted early on that PCV7 had differential immunological responses to VS, perhaps allowing certain MDR VS (such as $19 \mathrm{~F}$ ) to survive and co-circulate with non-VS (such as 19A) during PCV7 introduction in Canada [29]. "Capsule switch" has been described previously in SPN and would be the simplest genetic event to account for a $19 \mathrm{~F}$ to $19 \mathrm{~A}$ change $[12,30]$.

Brueggemann and colleagues carried out partial DNA sequencing at the capsule locus and demonstrated that recombination points likely lay distal to $p b p 1 a$ and $p b p 2 x$ based on sequence divergence between putative donor, recipient, and progeny strains [12]. This was an important demonstration of capsule switch from vaccine serotype 4 (ST695) to non-vaccine serotype 19A (ST199 and ST695) in the post-PCV7 era [12]. A similar comparison of $p b p 1 a$ and $p b p 2 x$ adjacent to the capsule locus from ST $19919 \mathrm{~A}$ (putative donor), ST 320 MDR 19A (putative progeny), and 19F (putative recipient) was undertaken in our study. These data demonstrate definitively that a homologous recombination event has occurred between donor and recipient with breakpoint within $p b p 2 x$ and flanking $p b p 1 a$, again reinforcing the fact that capsule switch can

Table 3: Amoxicillin minimum inhibitory concentration (MIC, top row in bold, $\mu \mathrm{g} / \mathrm{mL}$ ) values for multi-drug resistant (MDR) serotype I9A by sequence type (ST) $(n=97)$.

\begin{tabular}{lllllllllll}
\hline ST & $\mathbf{0 . 0 1}$ & $\mathbf{0 . 0 3}$ & $\mathbf{0 . 0 6}$ & $\mathbf{0 . 1 2}$ & $\mathbf{0 . 2 5}$ & $\mathbf{0 . 5}$ & $\mathbf{I}$ & $\mathbf{2}$ & $\mathbf{4}$ & $\geq \mathbf{8}$ \\
\hline 63 & & & 3 & & 2 & & & & & \\
199 & & & 2 & 11 & & & & & & \\
416 & 1 & 1 & 1 & & & & 1 & & & \\
230 & & & 3 & 7 & 1 & & & & & \\
230 & & & & & & 11 & 3 & 1 & & \\
320 & & & & & & & & I & I & 23 \\
2013 & & & & 1 & 1 & 1 & & & & \\
\hline
\end{tabular}

S, susceptible; I, intermediate; R, resistant; HR, highly resistant. 
Table 4: Erythromycin minimum inhibitory concentration (MIC, top row in bold, $\mu \mathrm{g} / \mathrm{mL}$ ) values for multi-drug resistant (MDR) serotype I9A by sequence type (ST) $(n=97)$.

\begin{tabular}{|c|c|c|c|c|c|c|c|c|c|c|c|c|c|}
\hline \multirow[b]{2}{*}{ ST } & \multirow[b]{2}{*}{0.03} & \multicolumn{3}{|c|}{$\mathbf{S}$} & \multicolumn{2}{|c|}{ I } & \multicolumn{7}{|c|}{$\mathbf{R}$} \\
\hline & & 0.06 & 0.12 & 0.25 & 0.5 & I & 2 & 4 & 8 & 16 & 32 & 64 & $\geq \mathbf{I} 28$ \\
\hline 63 & & & & & & & & & & I & 1 & 3 & \\
\hline 199 & & & 5 & & I & & & 2 & 4 & 1 & 1 & & \\
\hline 416 & & & 4 & & & & & & & & & & \\
\hline 230 & & I & 9 & & & I & & & & & & 1 & \\
\hline 276 & & & & & & & & & & & 2 & 12 & 1 \\
\hline 320 & & & & & & & & & & I & 4 & 20 & \\
\hline 2013 & & & 3 & & & & & & & & & & \\
\hline
\end{tabular}

S, susceptible; I, intermediate; R, resistant; HR, highly resistant.

occur between vaccine and non-vaccine serotypes thereby allowing "vaccine escape". This is a well documented strategy that SPN employs to enhance its fitness [31,32]. Of interest is the presence of the coding sequence of the vex operon (containing an efflux pump $\mathrm{ABC}$ co-transporter) associated with vancomycin tolerance adjacent to the capsule locus of 19A but located in its entirety outside of this region in 19F. While all strains in this study had an MIC of $\leq 0.5 \mu \mathrm{g} / \mathrm{mL}$ to vancomycin (our unpublished data), it is possible that the dysregulation of vex, an ABClike transporter, by uncoupling it from the rest of the operon, could lead to vancomycin MIC creep. Further evaluation of vancomycin MIC is required to determine the significance of this gene and its location within the capsule amongst MDR strains, especially as vancomycin remains the last line of defense against invasive gram positive infection [5].

In order to dissect the genome further, we undertook WGS comparing a representative isolate of ST320 MDR 19A and 19F. In keeping with the MLST data, near identity (99.7\% across the genome) was observed with 14,208 $(86 \%)$ shared SNPs, when compared to a reference strain R6, between MDR 19A and 19F ST320. Of note, MDR 19A was genetically closer (822 unique SNPs) to MDR 19F in

Table 5: Ciprofloxacin minimum inhibitory concentration (MIC, top row in bold, $\mu \mathrm{g} / \mathrm{mL}$ ) values for multi-drug resistant (MDR) serotype I9A by sequence type (ST) $(n=97)$.

\begin{tabular}{|c|c|c|c|c|c|c|c|c|c|}
\hline \multirow[b]{2}{*}{ ST } & \multicolumn{4}{|c|}{ S } & \multicolumn{2}{|l|}{ I } & \multicolumn{3}{|c|}{$\mathbf{R}$} \\
\hline & 0.12 & 0.25 & 0.5 & I & 2 & 4 & 8 & 16 & $\geq \mathbf{3 2}$ \\
\hline 63 & & & 0.5 & 4 & & & I & & \\
\hline 199 & & & 4 & 9 & I & & & & \\
\hline 199 & & & 2 & 1 & I & & & & \\
\hline 230 & & & I & 10 & & & & & \\
\hline 230 & & & & 14 & I & & & & \\
\hline 320 & & & I & 23 & & & & & I \\
\hline 2013 & & & 1 & 2 & & & & & \\
\hline
\end{tabular}

S, susceptible; I, intermediate; R, resistant; HR, highly resistant. this study than another serotype 19A from Hungary present in GenBank (9484 unique SNPs). These data reinforce that genetic relatedness is better indicated by MLST rather than serotype. Of the 822 unique SNPs identified in MDR 19A, some were in drug resistance markers, virulence factors, cell signaling, and key metabolic genes. Of these, 169 SNPs were non-synonymous. The presence of unique, non-synonymous SNPs inMDR 19A ST320 suggest that unique polymorphisms may also contribute to its success in the post-PCV7 era. The presence of mutations in metabolic genes raises the possibility of increased intrinsic fitness. However, we did not observe significant difference in growth kinetics between various STs associated with MDR 19A in liquid culture experiments (our unpublished data).

South Korea uniquely reported pre-PCV7 MDR 19A ST320. It remains unclear why MDR 19A would emerge without vaccine selection pressure against MDR 19F ST320. Canadian MDR 19A ST320 isolates used in this study were identical at key drug resistance markers to both US and Korean representative isolates suggesting a common source. There was, however, marked heterogeneity of transposon Tn2010 insertion sites within a geographic locale suggesting SPN undergoes rapid modification by this mode of genetic change. Furthermore, US isolates had unique mutations which confer high-level resistance to ceftriaxone and have been associated with other ST. Taken together, this suggests that MDR 19A emergence has been caused by distinct genetic events in different geographic locales rather than global spread of a single clone.

\section{Conclusions}

Our data provides evidence that MDR 19A ST320 is genetically derived from MDR 19F ST320 - based on MLST, conservation of SNPs across the genome, key drug resistance markers, and capsule locus structure. However, unique SNPs and heterogeneous transposition events also existed in MDR 19A ST320, suggesting that this strain has adapted and mutated away from a $19 \mathrm{~F}$ progenitor and is under 
Table 6: $\beta$-lactam resistance genes pbpla (penicillin), pbp2b (amoxicillin), and pbp2x (ceftriaxone) from reference strain R6 (Genbank AE007317), representative CBSN isolates, US isolates, and South Korean isolates.

\begin{tabular}{|c|c|c|c|c|c|c|c|c|c|c|}
\hline \multirow[b]{3}{*}{ Strain } & \multicolumn{10}{|c|}{ Changes in amino acids of conserved PBP sites } \\
\hline & \multicolumn{3}{|c|}{ pbp la } & \multicolumn{4}{|c|}{ pbp $2 x$} & \multicolumn{3}{|c|}{ pbp 2b } \\
\hline & $370-373$ & $428-432$ & $557-559$ & $337-340$ & $394-397$ & $400-401$ & $546-549$ & $385-388$ & $442-445$ & $614-616$ \\
\hline $19 F-14$ & SAMK & & KTG & SAMK & HSSN & MS & & & & \\
\hline MOH55 & SSMK & SRNVT & KTG & SAMK & HSSN & MS & VKSG & SVVK & SSNA & KTGTG \\
\hline MOH56 & SSMK & SRNVT & KTG & SAMK & HSSN & MS & VKSG & SVVK & SSNA & KTGTG \\
\hline MOHI47 & STMK & SRNVP & KTG & SAMK & HSSN & MT & LKSG & SVVK & SSNA & KTGTA \\
\hline МOH83 & SSMK & SRNVT & KTG & SAMK & HSSN & MS & VKSG & SVVK & SSNA & KTGTG \\
\hline MOH62 & SSMK & SRNVT & KTG & SAMK & HSSN & MS & VKSG & SVVK & SSNA & KTGTG \\
\hline Kor914 & SSMK & SRNVT & KTG & SAMK & HSSN & MS & VKSG & SVVK & SSNA & KTGTG \\
\hline Kor2I & SSMK & SRNVT & KTG & SAMK & HSSN & MS & VKSG & SVVK & SSNA & KTGTG \\
\hline Kor39 & SSMK & SRNVT & KTG & SAMK & HSSN & MS & VKSG & SVVK & SSNA & KTGTG \\
\hline PUII75 & SSMK & SRNVT & KTG & SAMK & HSSN & MS & VKSG & SVVK & SSNA & KTGTG \\
\hline PU6055 & SSMK & SRNVT & KTG & SAFK & HSSN & TS & VKSG & SVVK & SSNA & KTGTG \\
\hline
\end{tabular}

Serotype (SERO), sequence type (ST), clonal complex (CC) and MIC values ( $\mathrm{mg} / \mathrm{mL}$ ) for penicillin (PEN), ceftriaxone (CTX) and amoxicillin (AMOX) are indicated. Residues associated with resistance to $\beta$ lactams are in bold letters. Ceftriaxone MIC values for South Korea isolates were unavailable. Of note, Taiwan I9F-I4 (ST236) [Genbank E043521/E043522] retains residues similar to I9F and I9A in this study at certain residues of pbp/ $a$ and pbp2x. For the complete data set, including Tn2010-related resistance genes, see Additional file 3.

continuous selection pressure. MLST appears to be limited in explaining the genetic origins of a particular strain as it focuses only on seven housekeeping genes. We confirm that PCV7 vaccine selection pressure, antibiotic selection pressure, and SPN's propensity for genetic change appear to have created a "perfect storm" for MDR 19A emergence. Emergence of genetically heterogeneous MDR 19A appears to be occurring simultaneously in different geographic locales due to similar selection pressures.

Capsule switch events likely occur through genetic transformation in the nasopharynx of children co-infected with different ST of SPN. Of great concern to clinicians is that MDR 19A remains difficult to treat especially in the case of bacterial meningitis where few therapeutic options exist to penetrate the cerebrospinal fluid. If as with methicillin resistance Staphylococcus aureus (MRSA) vancomycin creep occurs (rising MIC values), newer antimicrobial agents will be needed. Furthermore, alternative vaccine strategies that target all serotypes of SPN (protein based vaccines) may be prudent in light of this organism's genetic lability and propensity for vaccine escape. A new 13-valent capsular polysaccharide vaccine (Wyeth, Madison, NJ) is slated for introduction and does include serotype 19A and may forestall its spread. Studies in the developing world are also required to fully understand the extent of the emergence of this strain.

\section{Methods}

\section{Source of isolates}

This work has been approved by the Institutional Review Board of Mt Sinai Hospital, Toronto, Canada. The Canadian Bacterial Surveillance Network is a volunteer group of private and hospital-affiliated laboratories from across
Canada which has performed surveillance for antibiotic resistance in Canadian isolates of $S$. pneumoniae since 1988 [33]. Isolates have been provided from a median of 50 laboratories annually which provide service to community and tertiary hospitals, as well as community clinics and doctors' offices. All ten provinces were represented in the sample collection. Laboratories, based on their size and catchment area, were asked to collect either the first 20 or 100 consecutive clinical isolates each year, as well as all sterile site isolates, from 1993 to 2008. In this database, 60\% were non-sterile and 40\% were sterile. Duplicate isolates from the same patient were excluded.

\section{Serotype and susceptibility testing}

Serotyping was done by the capsular swelling (quellung) test, using Danish antisera (State Serum Institute, Copenhagen, Denmark) [34]. In vitro susceptibility testing and interpretation was performed by broth microdilution according to Clinical and Laboratory Standards Institute guidelines [35]. The antimicrobial agents were supplied by their respective manufacturers or were purchased from Sigma (St. Louis, MO.). Multi-drug resistance (MDR) is defined as non-susceptible to penicillin plus any two other classes of antibiotics including macrolides, tetracyclines, fluoroquinolones, or trimethoprim-sulfa.

\section{Multi-locus sequence typing DNA sequencing, PCR, and whole genome sequencing}

MLST was performed according to the standard method described by Spratt and Enright http://www.mlst.net[36]. Briefly, seven housekeeping gene loci were sequenced bidirectionally, uploaded to the MLST website, and analyzed for sequence type and clonal complex associations based on the existing database. DNA sequencing of genes 


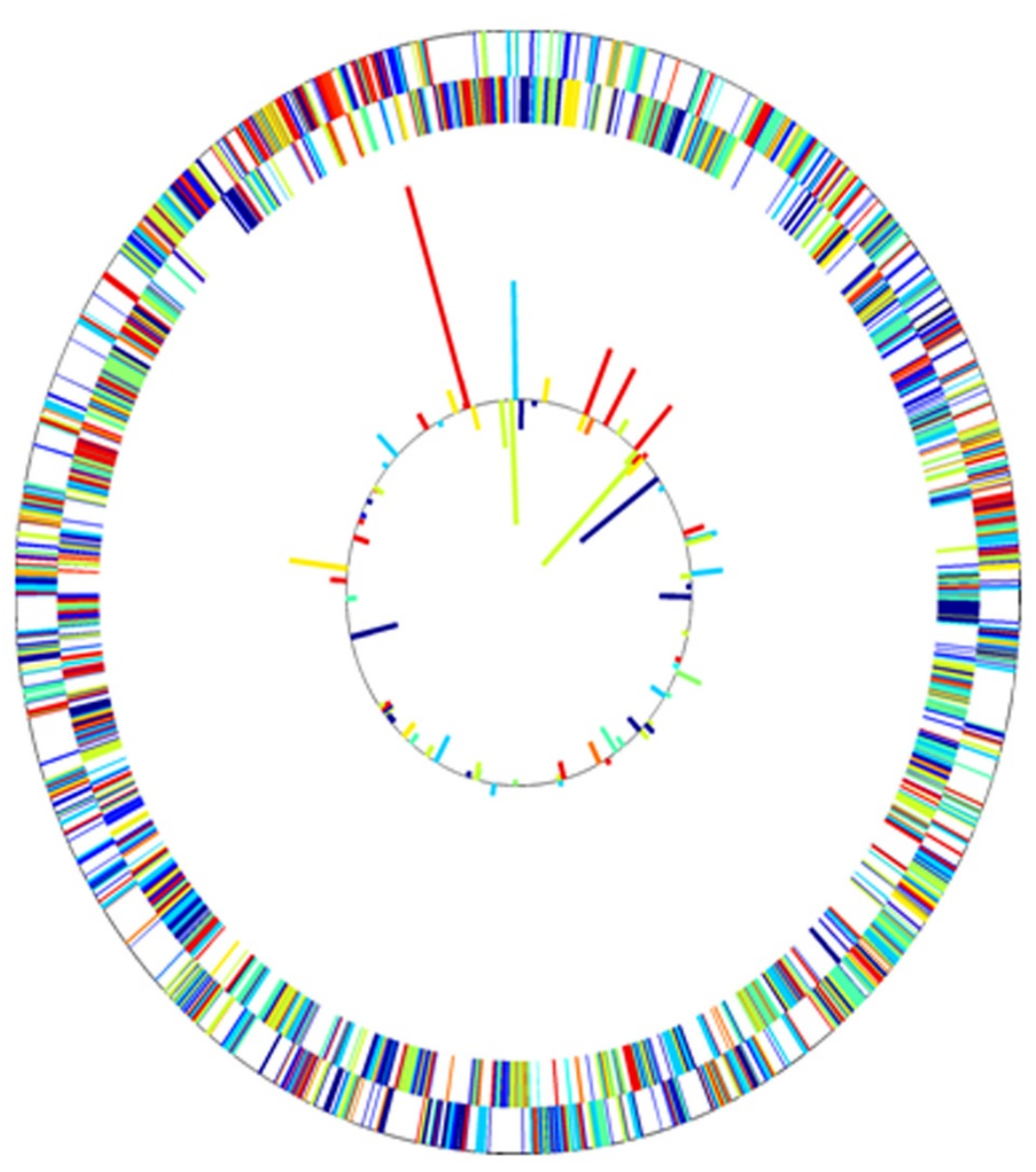

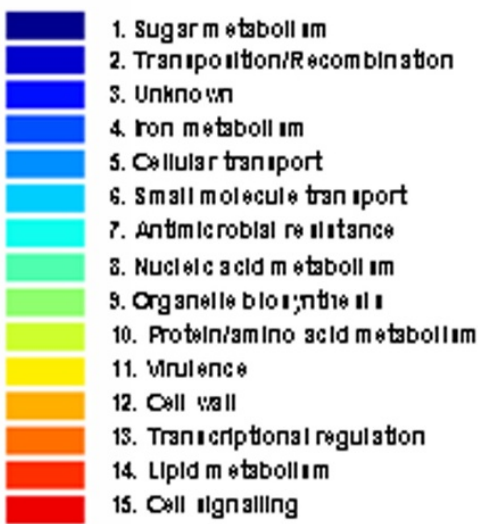

Figure 6

The whole genome sequence of a representative CBSN isolate of emergent multi-drug resistant serotype I9A ST320 (Genbank Accession GPID ACNU00000000) was compared to a representative isolate of I9F ST320 from the pre-vaccine era (Genbank Accession GPID ACNV00000000). The Solexa platform (Illumina Inc, San Diego, CA) was used for sequencing with greater than I00X coverage obtained throughout each genome. Depicted here is the whole genome of a representative MDR I9A ST320 in the post-vaccine era. The locations of proteins encoded on the leading and lagging strands are shown on the outer two rings. Gene ontology categories are color-coded. Of the internal ring, the outermost bars indicate SNPs identified in the leading strand and the innermost bars represent SNP identified in the lagging strand relative to MDR I9F ST320. The length of the bars is propostional to the number of SNPs. For detailed gene identification, location of capsule biosynthetic loci, other key alleles, comparison of I9A and I9F SNPs, as well as a mutation profile compared to reference strain R6 (Genbank AE0073 I7), see Additional file 4http://www.pillailab.com/suppdata/index.html.

associated with drug resistance $(p b p 1 a, p b p 2 b, p b p 2 x$, tetR and $\operatorname{erm} B$ ) was performed using a standard capillary gene sequencer from Applied Biosystems (Foster City, CA). The Solexa paired-end Sequencing Platform (Illumina, San Diego, CA) was used to generate reads of 50 to $75 \mathrm{bp}$ (with on average greater than $100 \mathrm{X}$ coverage for the genome) which were assembled using NextGene (SoftGenetics, State College, PA). [37]. Annotation of the genome was performed using the RAST (Rapid Annotation using
Subsystem Technology) server [38]. Additional file 9http:/ /www.pillailab.com/suppdata/index.html summarizes all polymerase chain reaction (PCR) and DNA sequencing primers and associated cycling parameters used in this study to confirm WGS findings at the capsule locus, resistance alleles, and transposon insertion sites. 


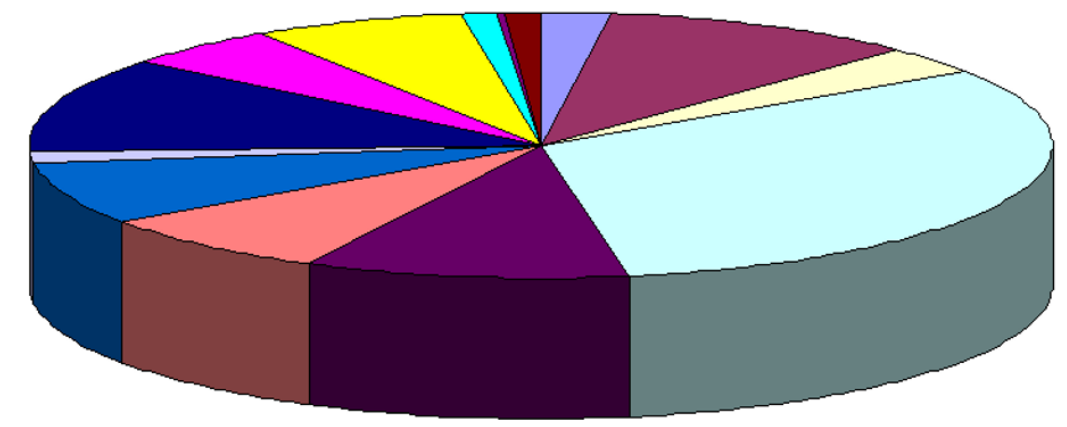

$\square$ Transcriptional regulation

Intergenic region

Transposition/Recombination

$\square$ Unknown

Protein metabolism

Nucleic acid metabolism

Sugar metabolism

Cell wall

Small molecule transport

$\checkmark$ Virulence

Cell Signalling

Antimicrobial resistance

Lipid metabolism

Organelle biosynthesis

Iron metabolism

\section{Figure 7}

Pie chart percentage breakdown by gene ontology classification (GenoList, Institut Pasteur, Paris) of single nucleotide polymorphisms (SNPs) belonging to MDR I9A ST320 relative to MDR I9F ST320.

\section{A}
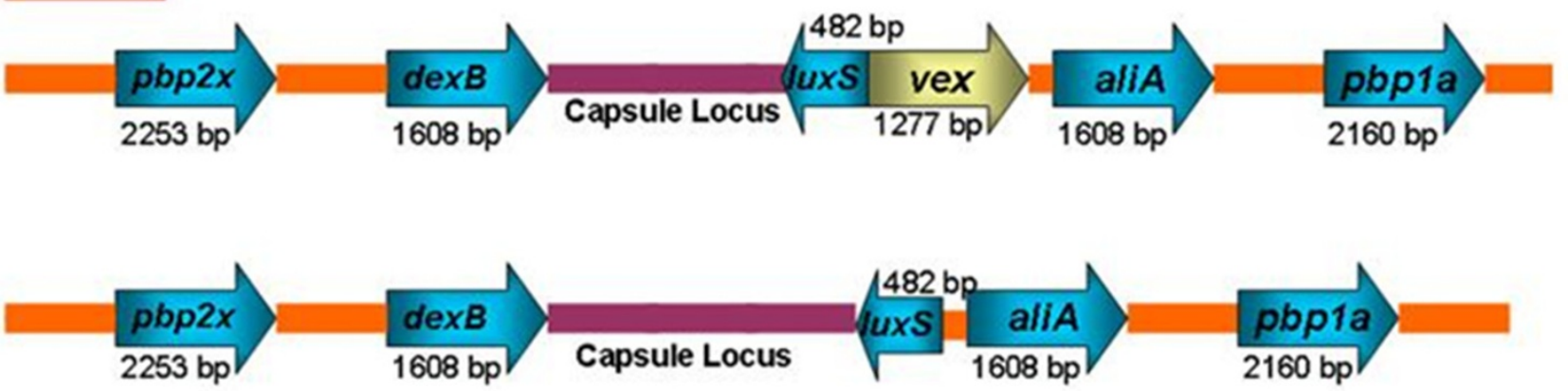

\section{$19 \mathrm{~F}$}

Figure 8

Alignment of the capsular biosynthetic loci between ST320 MDR I9A in the post-PCV7 introduction era and pre-PCV7 MDR I 9F. The capsule locus resides between genes aliA and dexB. The vex operon was present directly adjacent to the capsule locus of I9A but not I9F. Of note are the genes $p b p 2 x$ and $p b p / a$, responsible for penicillin resistance, that are present adjacent to the capsule locus. For a full image of the capsule locus and flanking regions please see Additional file 8http:l lwww.pillailab.com/suppdata/index.html. 


\section{Bioinformatic analysis}

eBURST analysis of MLST sequence data was performed as described on the MLST website http://www.mlst.net [39]. Genome assembly was performed using NextGene (SoftGenetics, State College, PA) and genome comparison using the Artemis Comparison Tool (Wellcome Trust Sanger Institute, Cambridge, UK). Gene ontology classification was achieved using GenoList (Institut Pasteur, Paris). Gene ontology categories were identified using AmiGO [21].

\section{Data Submission and Analysis}

This Whole Genome Shotgun project has been deposited at DDBJ/EMBL/GenBank under the accession ACNU00000000 (Streptococcus pneumoniae str. Canada MDR_19A identified in Ontario, Canada in 2007) and ACNV00000000 (Streptococcus pneumoniae str. Canada MDR_19F identified in Ontario, Canada in 2001). This paper describes the first versions of the whole genome for Canada MDR_19A (ACNU10000000) and MDR_19F (ACNV10000000). Detailed statistics about the genome sequences and the assembly, coverage and distribution reports as well as a summary of the SNP profiles is provided in Additional files 6 and 7. Novel MLST allele submission numbers are summarized in Additional file 2.

\section{Authors' contributions}

DRP and DS designed the study, analyzed the data, and wrote the manuscript. $\mathrm{AB}$ and RAP carried out data analysis and technical work. KK, AW, DJF, AM, KG, and DEL contributed to data analysis and manuscript review. DEL, $\mathrm{AM}$, and $\mathrm{KG}$, were responsible for providing the isolates, data anaylsis and manuscript review. All authors agree with the current version of the manuscript.

\section{Additional material}

\section{Additional file 1}

The complete MLST data set, summary statistics and eBURST figures. http://www.mlst.net. eBURST figures can be obtained by clicking on the hyperlinks. Figures are presented for MDR 19A, control 19A and $19 \mathrm{~F}$ isolates referred to in the text, as well as isolates based on era (pre-PCV7, PCV7, and post-PCV7). All supplementary data can also be accessed at the following website http://www.pillailab.com/suppdata/index.html Click here for file

[http://www.biomedcentral.com/content/supplementary/14712164-10-642-S1.XLS]

\section{Additional file 2}

New MLST sequence type and submission numbers identified in this study.

Click here for file

[http://www.biomedcentral.com/content/supplementary/14712164-10-642-S2.XLS]

\section{Additional file 3}

Complete data set for $\beta$ lactam (pbp), erythromycin (erm), and tetracycline (tet) resistance gene mutation alleles. Sequence analysis of Tn2010 insertion sites was based on primers designed from the WGS. A (+) indicated amplification and (-) no amplification by PCR for each isolate tested using primers derived from the WGS. These data demonstrated heterogeneity amongst MDR ST320 19A and MDR 19F isolates in the pre-PCV7 era with some common insertion points between serotypes. Tn2010 constituent genes ermB, mefA, tetM, and mega were highly conserved amongst post-PCV7 MDR 19A and pre-PCV7 MDR 19F. Click here for file

[http://www.biomedcentral.com/content/supplementary/14712164-10-642-S3.XLS]

\section{Additional file 4}

The annotated whole genome sequence data set for representative isolates of MDR 19A ST320. The first tab shows the full gene listing. To zoom in on a portion of the genome, click on the circular genome at the location of interest. The lower panel of the first tab depicts the entire capsule locus and flanking genes. The second tab gives the full mutation profile. The third tab gives the functional categories present in the genome. Click here for file

[http://www.biomedcentral.com/content/supplementary/14712164-10-642-S4.XLS]

\section{Additional file 5}

Mutation report of all single nucleotide polymorphisms, genomic location, and their gene ontology classification. Specific data for MDR $19 \mathrm{~F}$ (tab 1), 19A (tab 2), and both (tab 3), relative to reference strain $R 6$ (Genbank AE007317) are shown. Unique SNPs for 19F (tab 4) and 19A (tab 5) and subsequent analysis (tab 6) are alsolisted.

Click here for file

[http://www.biomedcentral.com/content/supplementary/14712164-10-642-S5.XLS]

\section{Additional file 6}

Whole genome statistics, SNP summary, distribution and coverage report of the genomes for MDR 19A

Click here for file

[http://www.biomedcentral.com/content/supplementary/14712164-10-642-S6.DOC]

\section{Additional file 7}

Whole genome statistics, SNP summary, distribution and coverage report of the genomes for MDR 19A

Click here for file

[http://www.biomedcentral.com/content/supplementary/14712164-10-642-S7.DOC] 


\begin{abstract}
Additional file 8
Sequence alignment of $\mathrm{pbp} 1 \mathrm{a}$ and $\mathrm{pbp} 2 \mathrm{x}$ for putative donor strain ST199 19A, putative recipient strain ST320 MDR 19F, and progeny strain ST320 MDR 19A. Yellow highlights sequence conservation between two of the three strain types. An arrow indicates the recombination point for $\mathrm{pbp} 2 \mathrm{x}$ where the conservation switches between strain types suggesting a recombination point. For $\mathrm{pbp} 1 \mathrm{a}$ no recombination point is identified within the gene suggesting that the point lies outside the gene. Heterologous and homologous single nucleotide polymorphisms (SNPs) are also present within $\mathrm{pbp} 2 \mathrm{x}$ but not $\mathrm{pbp} 1 \mathrm{a}$ (marked by arrows). A figure summarizing the genetic recombination event between donor, recipient and progeny strains is shown.

Click here for file

[http://www.biomedcentral.com/content/supplementary/14712164-10-642-S8.DOC]

\section{Additional file 9}

Complete set of primers and cycling conditions used for polymerase chain reaction (PCR) or DNA sequencing in this study.

Click here for file

[http://www.biomedcentral.com/content/supplementary/14712164-10-642-S9.XLS]
\end{abstract}

\section{Acknowledgements}

We thank the contributing laboratories that participate in the Canadian Bacterial Surveillance Network. We thank the staff of the Analytical Genetics Technology Centre (University Health Network, Toronto), Dr. David Alexander (Ontario Agency for Health Protection and Promotion) and Dr. David Guttman (Department of Cell and Systems Biology) for assistance and suggestions with the WGS analysis. We thank Dr J.H. Song of the Asian-Pacific Research Foundation for Infectious Diseases, Seoul, South Korea for providing isolates used in this study.

\section{References}

I. Dagan R, Klugman KP: Impact of conjugate pneumococcal vaccines on antibiotic resistance. Lancet Infect Dis 2008, 8:785-795.

2. Song JH, Baek JY, Cheong HS, Chung DR, Peck KR, Ko KS: Changes of serotype and genotype in Streptococcus pneumoniae isolates from a Korean hospital in 2007. Diagn Microbiol Infect Dis 2009, 63:27I-278.

3. Fenoll A, Granizo JJ, Aguilar L, Gimenez MJ, Aragoneses-Fenoll L, Hanquet G, Casal J, Tarrago D: Temporal trends of invasive Streptococcus pneumoniae serotypes and antimicrobial resistance patterns in Spain from 1979 to 2007. J Clin Microbiol 2009, 47:1012-1020.

4. Mahjoub-Messai F, Doit C, Koeck JL, Billard T, Evrard B, Bidet P, Hubans C, Raymond J, Levy C, Cohen R, Bingen E: Population snapshot of Streptococcus pneumoniae serotype 19A isolates before and after introduction of seven-valent pneumococcal Vaccination for French children. J Clin Microbiol 2009, 47:837-840

5. Ciccotelli WA, Poutanen SM, Alqahtani M, Morris SK, Cox P, Low DE, Pillai DR, Opavsky MA: A new twist on an old problem. A case of pediatric meningitis caused by multidrug-resistant Streptococcus pneumoniae serotype 19a. Pediatr Infect Dis J 2009, 28:74-75.

6. Jacobs MR, Good CE, Bajaksouzian S, Windau AR: Emergence of Streptococcus pneumoniae serotypes 19A, 6C, and 22F and serogroup 15 in Cleveland, Ohio, in relation to introduction of the protein-conjugated pneumococcal vaccine. Clin Infect Dis 2008, 47:1388-1395.

7. Amrine-Madsen H, Van EJ, Mera RM, Miller LA, Poupard JA, Thomas ES, Halsey WS, Becker JA, O'Hara FP: Temporal and spatial distribution of clonal complexes of Streptococcus pneumoniae isolates resistant to multiple classes of antibiotics in Bel- gium, 1997 to 2004. Antimicrob Agents Chemother 2008, 52:3216-3220.

8. Pelton SI, Huot H, Finkelstein JA, Bishop CJ, Hsu KK, Kellenberg J, Huang SS, Goldstein R, Hanage WP: Emergence of I9A as virulent and multidrug resistant Pneumococcus in Massachusetts following universal immunization of infants with pneumococcal conjugate vaccine. Pediatr Infect Dis J 2007, 26:468-472.

9. Dagan R: Impact of pneumococcal conjugate vaccine on infections caused by antibiotic-resistant Streptococcus pneumoniae. Clin Microbiol Infect 2009, 15(Suppl 3): 16-20.

10. Moore MR, Gertz RE Jr, Woodbury RL, Barkocy-Gallagher GA, Schaffner W, Lexau C, Gershman K, Reingold A, Farley M, Harrison LH, Hadler JL, Bennett NM, Thomas AR, McGee L, Pilishvili T, Brueggemann AB, Whitney CG, Jorgensen JH, Beall B: Population snapshot of emergent Streptococcus pneumoniae serotype I9A in the United States, 2005. J Infect Dis 2008, 197: 1016-1027.

II. Bae S, Lee K: Distribution of capsular serotypes and macrolide resistance mechanisms among macrolide-resistant Streptococcus pneumoniae isolates in Korea. Diagn Microbiol Infect Dis 2009, 63:213-216.

12. Brueggemann AB, Pai R, Crook DW, Beall B: Vaccine escape recombinants emerge after pneumococcal vaccination in the United States. PLoS Pathog 2007, 3: el 68.

13. Pai R, Moore MR, Pilishvili T, Gertz RE, Whitney CG, Beall B: Postvaccine genetic structure of Streptococcus pneumoniae serotype 19A from children in the United States. J Infect Dis 2005, 192:1988-1995.

14. Choi EH, Kim SH, Eun BW, Kim SJ, Kim NH, Lee J, Lee HJ: Streptococcus pneumoniae serotype 19A in children, South Korea. Emerg Infect Dis 2008, 14:275-28I.

15. Zhanel GG, Wang X, Nichol K, Nikulin A, Wierzbowski AK, Mulvey $M$, Hoban DJ: Molecular characterisation of Canadian paediatric multidrug-resistant Streptococcus pneumoniae from 1998-2004. Int J Antimicrob Agents 2006, 28:465-47I.

16. McClure CA, Ford MW, Wilson JB, Aramini J]: Pneumococcal conjugate vaccination in Canadian infants and children younger than five years of age: Recommendations and expected benefits. Can J Infect Dis Med Microbiol 2006, 17:19-26.

17. Hsieh YC, Chang KY, Huang YC, Lin HC, Ho YH, Huang LM, Hsueh PR: Clonal spread of highly beta-lactam-resistant Streptococcus pneumoniae isolates in Taiwan. Antimicrob Agents Chemother 2008, 52:2266-2269.

18. Farrell DJ, Morrissey I, Bakker S, Morris L, Buckridge S, Felmingham D: Molecular epidemiology of multiresistant Streptococcus pneumoniae with both erm(B)- and mef(A)-mediated macrolide resistance. J Clin Microbiol 2004, 42:764-768.

19. Grebe T, Hakenbeck R: Penicillin-binding proteins $\mathbf{2 b}$ and $2 \mathrm{x}$ of Streptococcus pneumoniae are primary resistance determinants for different classes of beta-lactam antibiotics. Antimicrob Agents Chemother 1996, 40:829-834.

20. Jacobs MR, Good CE, Beall B, Bajaksouzian S, Windau AR, Whitney CG: Changes in serotypes and antimicrobial susceptibility of invasive Streptococcus pneumoniae strains in Cleveland: a quarter century of experience. J Clin Microbiol 2008, 46:982-990.

21. Carbon S, Ireland A, Mungall C], Shu S, Marshall B, Lewis S: AmiGO: online access to ontology and annotation data. Bioinformatics 2009, 25:288-289.

22. Bentley SD, Aanensen DM, Mavroidi A, Saunders D, Rabbinowitsch $E$, Collins M, Donohoe K, Harris D, Murphy L, Quail MA, Samuel G, Skovsted IC, Kaltoft MS, Barrell B, Reeves PR, Parkhill J, Spratt BG: Genetic analysis of the capsular biosynthetic locus from all 90 pneumococcal serotypes. PLoS Genet 2006, 2:e31.

23. Morona JK, Morona R, Paton JC: Analysis of the 5 ' portion of the type I9A capsule locus identifies two classes of cpsC, cpsD, and cpsE genes in Streptococcus pneumoniae. J Bacteriol 1999, | 81:3599-3605

24. Haas W, Sublett J, Kaushal D, Tuomanen El: Revising the role of the pneumococcal vex-vncRS locus in vancomycin tolerance. | Bacteriol 2004, | 86:8463-847|.

25. Pichichero ME, Casey JR: Emergence of a multiresistant serotype 19A pneumococcal strain not included in the 7-valent conjugate vaccine as an otopathogen in children. JAMA 2007, 298: 1772-1778.

26. Siira L, Rantala M, Jalava J, Hakanen AJ, Huovinen P, Kaijalainen T, Lyytikainen $O$, Virolainen $A$ : Temporal trends of antimicrobial 
resistance and clonality of invasive Streptococcus pneumoniae isolates in Finland, 2002 to 2006. Antimicrob Agents Chemother 2009, 53:2066-2073.

27. Richter SS, Heilmann KP, Dohrn CL, Riahi F, Beekmann SE, Doern GV: Changing epidemiology of antimicrobial-resistant Streptococcus pneumoniae in the United States, 2004-2005. Clin Infect Dis 2009, 48:e23-e33.

28. Mavroidi A, Paraskakis I, Pangalis A, Kirikou E, Charisiadou A, Athanasiou T, Tassios PT, Tzouvelekis LS: Spread of the Streptococcus pneumoniae TaiwanI9F-14 clone among children in Greece. Clin Microbiol Infect 2007, I3:1213-1216.

29. Zangwill KM, Greenberg DP, Chiu CY, Mendelman P, Wong VK, Chang SJ, Partridge S, Ward JI: Safety and immunogenicity of a heptavalent pneumococcal conjugate vaccine in infants. Vaccine 2003, 2 I: 1894-1900.

30. Brugger SD, Hathaway LJ, Muhlemann K: Streptococcus pneumoniae strain co-colonization in the nasopharynx. J Clin Microbiol 2009, 47: I 750-6.

31. Temime L, Boelle PY, Opatowski L, Guillemot D: Impact of capsular switch on invasive pneumococcal disease incidence in a vaccinated population. PLoS One 2008, 3:e3244.

32. Dillard JP, Caimano M, Kelly T, Yother J: Capsules and cassettes: genetic organization of the capsule locus of Streptococcus pneumoniae. Dev Biol Stand 1995, 85:261-265.

33. Chen DK, McGeer A, de Azavedo JC, Low DE: Decreased susceptibility of Streptococcus pneumoniae to fluoroquinolones in Canada. Canadian Bacterial Surveillance Network. N Engl J Med 1999, 341:233-239.

34. Lauer BA, Reller LB: Serotypes and penicillin susceptibility of pneumococci isolated from blood. J Clin Microbiol 1980, I I:242-244.

35. Clinical and Laboratory Standards Institute Performance standards for antimicrobial susceptibility testing: Eighteenth informational supplement. In Document MI00-S I 8 edition Wayne (PA): The Institute; 2008.

36. Enright MC, Spratt BG: A multilocus sequence typing scheme for Streptococcus pneumoniae: identification of clones associated with serious invasive disease. Microbiology I998, I44(Pt I I):3049-3060.

37. Hillier LW, Marth GT, Quinlan AR, Dooling D, Fewell G, Barnett D, Fox P, Glasscock Jl, Hickenbotham M, Huang W, Magrini VJ, Richt RJ, Sander SN, Stewart DA, Stromberg M, Tsung EF, Wylie T, SchedI T, Wilson RK, Mardis ER: Whole-genome sequencing and variant discovery in C. elegans. Nat Methods 2008, 5: I83-I88.

38. Aziz RK, Bartels $D$, Best AA, Delongh $M$, Disz $T$, Edwards RA, Formsma K, Gerdes S, Glass EM, Kubal M, Meyer F, Olsen GJ, Olson R, Osterman AL, Overbeek RA, McNeil LK, Paarmann D, Paczian T, Parrello B, Pusch GD, Reich C, Stevens R, Vassieva O, Vonstein V, Wilke A, Zagnitko O: The RAST Server: rapid annotations using subsystems technology. BMC Genomics 2008, 9:75.

39. Feil EJ, Li BC, Aanensen DM, Hanage WP, Spratt BG: eBURST: inferring patterns of evolutionary descent among clusters of related bacterial genotypes from multilocus sequence typing data. J Bacteriol 2004, 186:1518-1530.
Publish with Bio Med Central and every scientist can read your work free of charge

"BioMed Central will be the most significant development for disseminating the results of biomedical research in our lifetime. "

Sir Paul Nurse, Cancer Research UK

Your research papers will be:

- available free of charge to the entire biomedical community

- peer reviewed and published immediately upon acceptance

- cited in PubMed and archived on PubMed Central

- yours - you keep the copyright
BioMedcentral 